\title{
39. Benchmark solutions of the free vibration of simply supported laminated composite plates
}

\author{
Xinwei Wang \\ State Key Laboratory of Mechanics and Control of Mechanical Structures, Nanjing University of \\ Aeronautics and Astronautics, No. 29 Yudao Street, Nanjing, 210016, China \\ E-mail:wangx@nuaa.edu.cn
}

Received 28 September 2017; accepted 10 October 2017

DOI https://doi.org/10.21595/mme.2017.19257

Check for updates

\begin{abstract}
Due to the high specific strength and stiffness, laminated composite plates, especially mid-plane symmetric laminated composite plates, are frequently used as structural component in aeronautical and aerospace engineering. Since obtaining analytic solutions are difficult even for simply supported mid-plane symmetric laminated composite plates, numerical methods have to be used to obtain approximate solutions. To evaluate various numerical methods, benchmark solutions are needed. In this article, highly accurate frequencies of simply supported angle-ply mid-plane symmetric laminated composite plates with two sets of equivalent material properties are obtained by the modified differential quadrature method and presented to serve as the benchmark solutions.
\end{abstract}

Keywords: laminated composite plate, equivalent material properties; benchmark solution, free vibration; modified differential quadrature method.

\section{Introduction}

Due to the high specific strength and stiffness, laminated composite plates are frequently used as structural component in aeronautical and aerospace engineering. Their static, buckling and free vibration behavior is of important to the designers and thus has been received great attentions $[1,2]$. Among various types of laminations, the mid-plane symmetric laminates are widely used in practice. The de-coupling of in-plane and out-of-plane deformation makes the production of a flat plate as well as analysis much simpler than the general laminations.

In free vibration analysis, the laminated composite plates are usually equivalent to anisotropic plates. Analytical solutions are rarely available even for rectangular anisotropic plates with simple supported boundary conditions. Therefore, various approximate approaches [1-4] and numerical methods [5-9] have been employed for solutions.

In literature, two equivalent ways of expressing the material properties are commonly used. Take the E-glass/epoxy (E/E) material as an example, the material properties expressed in one way, called the material system I (MS-I), are $E_{1}=60.7 \mathrm{GPa}, E_{2}=24.8 \mathrm{GPa}, G_{12}=12.0 \mathrm{GPa}$ and $v_{12}=0.23[3,8,10]$, and the material properties expressed in the other way, called the material system II (MS-II), are $E_{1} / E_{2}=2.45, G_{12} / E_{2}=0.48$ and $v_{12}=0.23[4,6,7]$. Researchers often do not distinguish one set of material properties from the other since they are regarded equivalent. The choice mainly depends on their personal preference. In references $[4,6,7]$, the results are obtained based on the MS-II, but compared with the upper bound solutions with the MS-I [3]. In reference [8], the material properties of MS-I are given, but the data are actually obtained with the ones with MS-II. Occasionally this might cause mis-understanding to the readers, although it is not difficult to tell that MS-II is actually used in their calculation by looking at the exact solutions of special orthotropic rectangular plates, since the exact solutions for the two sets of equivalent material systems are slightly different and the corresponding Ritz solutions of special orthotropic plates reported in [3] are also exact solutions [10]. The difference in solutions for the laminated composite plates with the two sets of equivalent material properties is really small and negligible from the practical point of view.

From the computational point of view, however, the small difference may be important in testing the accuracy and efficiency of new numerical methods. Very accurate benchmark solutions 
are required in such cases. The data reported in $[3,4]$ are not very accurate due to either the lower rate of convergence of the method or the extra constraints implicitly enforced in the test functions [11]. More terms in the series of the test functions are needed to obtained solutions with higher accuracy by using the Ritz method.

The primary objective of this paper is to provide highly accurate benchmark frequencies for simply supported square laminated composite plates with two sets of equivalent material properties. The modified differential quadrature method proposed by the author is used to obtain accurate solutions. The slight difference in the frequencies of the mid-plane symmetric laminates with two sets of equivalent material systems is clearly demonstrated.

\section{Basic equations and solution procedures}

\subsection{Governing equation and expression of boundary condition}

Denote the length, width and total thickness of the rectangular laminated composite plate by $a, b$, and $h$. The governing equation for the free vibration analysis of a mid-plane symmetric laminated composite plate is given by:

$\bar{D}_{11} \frac{\partial^{4} w}{\partial x^{4}}+4 \bar{D}_{16} \frac{\partial^{4} w}{\partial x^{3} \partial y}+2\left(\bar{D}_{12}+2 \bar{D}_{66}\right) \frac{\partial^{4} w}{\partial x^{2} \partial y^{2}}+4 \bar{D}_{26} \frac{\partial^{4} w}{\partial x \partial y^{3}}+\bar{D}_{22} \frac{\partial^{4} w}{\partial y^{4}}=\rho h \omega^{2} w$,

where $\bar{D}_{i j}$ are the effective bending and twisting stiffness [12], $w(x, y)$ is the deflection, $\rho$ and $\omega$ are the mass density and circular frequency, respectively.

The expressions of simply supported boundary conditions are:

$\begin{cases}w=0, & M_{x}=0(x=0, a) \\ w=0, & M_{y}=0(y=0, b)\end{cases}$

where the expressions of bending moments $M_{x}$ and $M_{y}$ are:

$\left\{\begin{array}{l}M_{x}=\bar{D}_{11} \frac{\partial^{2} w}{\partial x^{2}}+2 \bar{D}_{16} \frac{\partial^{2} w}{\partial x \partial y}+\bar{D}_{12} \frac{\partial^{2} w}{\partial y^{2}} \\ M_{y}=\bar{D}_{12} \frac{\partial^{2} w}{\partial x^{2}}+2 \bar{D}_{26} \frac{\partial^{2} w}{\partial x \partial y}+\bar{D}_{22} \frac{\partial^{2} w}{\partial y^{2}}\end{array}\right.$

\subsection{Modified differential quadrature method and solution procedures}

For completeness considerations, the modified differential quadrature method (modified DQM) and solution procedures are briefly introduced.

Denote $N_{x}$ and $N_{y}$ the numbers of grid points in $x$ and $y$ directions, and $\left(x_{i}, y_{j}\right)$ $\left(i=1,2, \ldots, N_{x} ; j=1,2, \ldots, N_{y}\right)$ the grid points. In the modified DQM, two additional derivative degrees of freedom at end points are introduced by using the method of modification of weighting coefficient-3 (MMWC-3) proposed by the author [9].

For simplicity and demonstration of the method, take a one-dimensional problem as an example. In the ordinary differential quadrature method, the first order derivative of the solution $w(x)$ with respect to $x$ at grid point $x_{i}$ is approximated as:

$\left.\frac{d w}{d x}\right|_{x=x_{i}}=\sum_{j=1}^{N_{x}} A_{i j}^{x} w_{j}, \quad\left(i=1,2, \ldots, N_{x}\right)$, 
where $A_{i j}^{x}$ is called the weighting coefficient, which can be explicitly computed by:

$$
A_{i j}^{x}=\left\{\begin{array}{l}
\prod_{k=1, k \neq i, j}^{N_{x}} \frac{x_{i}-x_{k}}{\prod_{k=1, k \neq j}^{N_{x}}\left(x_{j}-x_{k}\right)}, \quad(i \neq j), \\
\sum_{k=1, k \neq i}^{N_{x}} \frac{1}{\left(x_{i}-x_{k}\right)}, \quad(i=j),
\end{array} \quad\left(i, j=1,2, \ldots, N_{x}\right) .\right.
$$

To apply multiple boundary conditions rigorously, two additional degrees of freedom (DOFs) are introduced during formulation the weighting coefficient of the second order derivatives at two end points by using the MMWC-3 [9], namely:

$$
\begin{gathered}
\left.\frac{d^{2} w}{d x^{2}}\right|_{x=x_{i}}=\left.\sum_{k=1}^{N_{x}} A_{i k}^{x} \frac{d w}{d x}\right|_{x=x_{k}}=\sum_{k=2}^{N_{x}-1} A_{i k}^{x} \sum_{j=1}^{N_{x}} A_{k j}^{x} w_{j}+A_{i 1}^{x} w_{1}^{\prime}+A_{i N}^{x} w_{N}^{\prime} \\
=\sum_{j=1}^{N_{x}} \tilde{B}_{i j}^{x} w_{j}+A_{i 1}^{x} w_{1}^{\prime}+A_{i N}^{x} w_{N}^{\prime}=\sum_{j=1}^{N+2} \tilde{B}_{i j}^{x} \delta_{j}, \quad\left(i=1, N_{x}\right),
\end{gathered}
$$

where $\delta_{j}=w_{j}\left(j=1,2, \ldots, N_{x}\right), \delta_{N+1}=w_{1}^{\prime}, \delta_{N+2}=w^{\prime}{ }_{N}, \tilde{B}_{i(N+1)}^{x}=A_{i 1}^{x}, \tilde{B}_{i(N+2)}^{x}=A_{i N}^{x}$.

At all inner points, the weighting coefficients of the second order derivative are the same as the ordinary DQM, namely:

$$
\left\{\begin{array}{l}
\tilde{B}_{i j}^{x}=B_{i j}^{x}=\sum_{k=1}^{N_{x}} A_{i k}^{x} A_{k j}^{x}, \quad\left(i=2,3, \ldots, N_{x}-1, \quad j=1,2, \ldots, N_{x}\right), \\
\tilde{B}_{i j}^{x}=0, \quad\left(i=2,3, \ldots, N_{x}-1, \quad j=N_{x}+1, N_{x}+2\right), \\
B_{i j}^{x}=\sum_{k=1}^{N_{x}} A_{i k}^{x} A_{k j}^{x}, \quad \tilde{B}_{i j}^{x}=\sum_{k=2}^{N_{x}-1} A_{i k}^{x} A_{k j}^{x}, \quad\left(i=1, N_{x}, \quad j=1,2, \ldots, N_{x}\right) .
\end{array}\right.
$$

In the modified DQM, the weighting coefficients of the third and the fourth order derivatives, denoted by $\tilde{C}_{i j}^{x}, \widetilde{D}_{i j}^{x}$ are computed by:

$\tilde{C}_{i j}^{x}=\sum_{k=1}^{N_{x}} A_{i k}^{x} \tilde{B}_{k j}^{x}, \quad\left(i=1,2, \ldots, N_{x}, j=1,2, \ldots, N_{x}+2\right)$,
$\widetilde{D}_{i j}^{x}=\sum_{k=1}^{N_{x}} B_{i k}^{x} \tilde{B}_{k j}^{x}, \quad\left(i=1,2, \ldots, N_{x}, j=1,2, \ldots, N_{x}+2\right)$.

The weighting coefficients of the first to fourth-order derivatives with respect to $y$ can be calculated in a similar way, simply replacing $x$ and $N_{x}$ in Eq. (5) to Eq. (9) by $y$ and $N_{y}$. Since only square plates $(a=b)$ are considered, thus $N_{x}=N_{y}=N$. In terms of the modified differential quadrature (DQ), the bending moments at corresponding boundary points can be expressed as: 


$$
\begin{gathered}
\left(M_{x}\right)_{i l}=\bar{D}_{11} \sum_{k=1}^{N+2} \tilde{B}_{i k}^{x} \widetilde{w}_{k l}+2 \bar{D}_{16} \sum_{j=1}^{N} \sum_{k=1}^{N} A_{i j}^{x} A_{l k}^{y} \widetilde{w}_{j k}+\bar{D}_{12} \sum_{k=1}^{N+2} \tilde{B}_{l k}^{y} \widetilde{w}_{i k}, \\
(i=1, N, \quad l=1,2, \ldots, N) \\
\left(M_{y}\right)_{i l}=\bar{D}_{12} \sum_{k=1}^{N+2} \tilde{B}_{i k}^{x} \widetilde{w}_{k l}+2 \bar{D}_{26} \sum_{j=1}^{N} \sum_{k=1}^{N} A_{i j}^{x} A_{l k}^{y} \widetilde{w}_{j k}+\bar{D}_{22} \sum_{k=1}^{N+2} \tilde{B}_{l k}^{y} \widetilde{w}_{i k} \\
(i=1,2, \ldots, N, \quad l=1, N) .
\end{gathered}
$$

In terms of the DQ, the governing equation at all grid points can be expressed as:

$$
\begin{aligned}
& \bar{D}_{11} \sum_{k=1}^{N+2} \widetilde{D}_{i k}^{x} \bar{w}_{k l}+4 \bar{D}_{16} \sum_{j=1}^{N+2} \sum_{k=1}^{N} \tilde{C}_{i j}^{x} A_{l k}^{y} \bar{w}_{j k}+2\left(\bar{D}_{12}+2 \bar{D}_{66}\right) \sum_{j=1}^{N} \sum_{k=1}^{N} B_{i j}^{x} B_{l k}^{y} \bar{w}_{j k} \\
& +4 \bar{D}_{26} \sum_{j=1}^{N} \sum_{k=1}^{N+2} A_{i j}^{x} \tilde{C}_{l k}^{y} \bar{w}_{j k}+\bar{D}_{22} \sum_{k=1}^{N+2} \widetilde{D}_{l k}^{y} \bar{w}_{i k}=\rho h \omega^{2} w_{i l}, \quad(i, l=1,2, \ldots, N),
\end{aligned}
$$

where superscripts $x$ and $y$ mean that the weighting coefficients of the corresponding derivatives are taken with respect to $x$ and $y, \bar{w}_{i k}$ contains the deflection $w_{i l}$ as well as the first-order derivative with respect to $x$ or $y$ along boundary points, introduced by the method of modification of weighting coefficient-3 (MMWC-3), $\widetilde{w}_{k l}, \widetilde{w}_{j k}$ and $\widetilde{w}_{i k}$ are only a part of $\bar{w}_{i k}$. There are $(N+2) \times(N+2)-4$ degrees of freedom (DOFs) in total. From Eq. (7), it is clearly seen that $B_{i j}^{x}(i=1, N)$ are different from $\tilde{B}_{i j}^{x}(i=1, N)$, and $B_{l k}^{y}(l=1, N)$ are different from $\tilde{B}_{l k}^{y}$ $(l=1, N)$.

The bending moment equation is placed at the position where the DOF of the first-order derivative with respect to $x$ or $y$ at corresponding boundary point is. Enforcing the simply supported boundary conditions rigorously yields following partitioned matrix equations, namely:

$$
\left[\begin{array}{ll}
{\left[K_{\alpha \alpha}\right]} & {\left[K_{\alpha \beta}\right]} \\
{\left[K_{\beta \alpha}\right]} & {\left[K_{\beta \beta}\right]}
\end{array}\right]\left\{\begin{array}{l}
\left\{w_{\alpha}\right\} \\
\left\{w_{\beta}\right\}
\end{array}\right\}=\Omega^{2}\left[\begin{array}{ll}
{[I]} & {[0]} \\
{[0]} & {[0]}
\end{array}\right]\left\{\begin{array}{l}
\left\{w_{\alpha}\right\} \\
\left\{w_{\beta}\right\}
\end{array}\right\}
$$

where $\Omega=\omega a^{2} \sqrt{\rho h / D_{0}}$ is called the frequency parameter, $D_{0}=E_{1} h^{3} /\left[12\left(1-v_{12} v_{21}\right)\right], E_{1}$, $v_{12}$ and $v_{21}$ are the modulus of elasticity in the fiber direction, as well as the major and minor Poisson's ratios, respectively. The vector $\left\{w_{\alpha}\right\}$ contains only the non-zero DOFs of the deflection at all inner grid points and its dimension is $(N-2) \times(N-2)$.

After eliminating $\left\{w_{\beta}\right\}$, Eq. (13) can be rewritten in the following matrix equation:

$[\bar{K}]\left\{w_{\alpha}\right\}=\Omega^{2}[I]\left\{w_{\alpha}\right\}$,

where $[\bar{K}]=\left[K_{\alpha \alpha}-K_{\alpha \beta} K_{\beta \beta}^{-1} K_{\beta \alpha}\right]$.

Solving Eq. (14) by a standard eigen-solver yields the frequency parameters.

To achieve the fastest rate of convergence and obtain reliable and accurate solutions, following grid points are used in the modified DQM:

$x_{k}=y_{k}=\frac{a\left[1-\cos \left(\frac{(k-1) \pi}{N-1}\right)\right]}{2},(k=1,2, \ldots, N, \quad a=b)$.

The exact frequency parameters $(\Omega)$ for especially orthotropic rectangular plates can be calculated analytically by $[1,3]$ : 


$$
\begin{gathered}
\Omega_{\text {exact }}=\omega a^{2} \sqrt{\frac{\rho h}{D_{0}}}=\pi^{2} \sqrt{\frac{\bar{D}_{11}}{D_{0}} m^{4}+2\left(\frac{\bar{D}_{12}}{D_{0}}+2 \frac{\bar{D}_{66}}{D_{0}}\right)\left(\frac{a}{b}\right)^{2} m^{2} n^{2}+\frac{\bar{D}_{22}}{D_{0}}\left(\frac{a}{b}\right)^{2} n^{4},} \\
(m, n=1,2, \ldots),
\end{gathered}
$$

where $m$ and $n$ are the half wave number of the vibration mode in $x$ and $y$ directions, respectively.

\section{Results and discussion}

Three materials of lamina, i.e., E-glass/epoxy (E/E), Boron/epoxy (B/E) and Graphite/epoxy $(\mathrm{G} / \mathrm{E})$, are considered. The material parameters directly taken from $[3,4]$ are listed in Table 1 . For each material, two sets of equivalent material constants are given. Among the three materials, Graphite/epoxy exhibits the highest anisotropy, since $E_{1} / E_{2}$ is the largest.

Table 1. Material property of two sets of equivalent material constants

\begin{tabular}{|c|c|c|c|c|c|c|c|}
\hline \multirow{2}{*}{ Materials } & \multicolumn{4}{|c|}{ Material system I (MS-I) [3] } & \multicolumn{3}{c|}{ Material system II (MS-II) [4] } \\
\cline { 2 - 8 } & $E_{1}(\mathrm{GPa})$ & $E_{2}(\mathrm{GPa})$ & $G_{12}(\mathrm{GPa})$ & $v_{12}$ & $E_{1} / E_{2}$ & $G_{12} / E_{2}$ & $v_{12}$ \\
\hline $\mathrm{E} / \mathrm{E}$ & 60.7 & 24.8 & 12.0 & 0.23 & 2.45 & 0.48 & 0.23 \\
\hline $\mathrm{B} / \mathrm{E}$ & 209. & 19.0 & 6.40 & 0.21 & 11.0 & 0.34 & 0.21 \\
\hline $\mathrm{G} / \mathrm{E}$ & 138. & 8.96 & 7.10 & 0.30 & 15.4 & 0.79 & 0.30 \\
\hline
\end{tabular}

Denote $\theta$ the fiber orientation angle. Four angles, i.e., $\theta=0^{\circ}, 15^{\circ}, 30^{\circ}$ and $45^{\circ}$, are considered. The relative bending-twisting coupling coefficients $D_{16} / D_{0}$ and $D_{26} / D_{0}$, which reflect the degrees of anisotropy, are listed in Table 2.

Table 2. Relative bending-twisting coefficients of angle-ply $(\theta /-\theta / \theta)$ laminated plates

\begin{tabular}{|c|c|c|c|c|c|c|}
\hline \multirow{2}{*}{$\theta^{\circ}$} & \multicolumn{2}{|c|}{ E-glass/epoxy (E/E) } & \multicolumn{2}{c|}{ Boron/epoxy (B/E) } & \multicolumn{2}{c|}{ Graphite/epoxy (G/E) } \\
\cline { 2 - 7 } & $D_{16} / D_{0}$ & $D_{26} / D_{0}$ & $D_{16} / D_{0}$ & $D_{26} / D_{0}$ & $D_{16} / D_{0}$ & $D_{26} / D_{0}$ \\
\hline 0 & 0.000000 & 0.000000 & 0.000000 & 0.000000 & 0.000000 & 0.000000 \\
\hline 15 & 0.122312 & 0.012555 & 0.214391 & 0.012882 & 0.205801 & 0.027967 \\
\hline 30 & 0.176432 & 0.079666 & 0.297578 & 0.096069 & 0.291366 & 0.113533 \\
\hline 45 & 0.147858 & 0.147858 & 0.227273 & 0.227273 & 0.233768 & 0.233768 \\
\hline
\end{tabular}

It is seen that $D_{16} / D_{0}$ is the largest when $\theta=30^{\circ}$. This perhaps is the reason why the convergence study is performed for $\theta=30^{\circ}$ in [3], since the higher the anisotropy, the lower the rate of convergence for various approximate and numerical methods. Although $D_{16} / D_{0}$ is the second largest when $\theta=45^{\circ}$, however, $D_{26} / D_{0}$ is the largest. Thus, convergence studies are performed for both $\theta=30^{\circ}$ and $\theta=45^{\circ}$ in present investigations. Corresponding results are listed in Table 3 and Table 4, respectively. Mid-plane symmetric angle-ply square plates with all edges simply supported, denoted by SSSS, are investigated.

From Table 3 and Table 4, it is clearly seen that the rate of convergence of the DQM is high. The rate of convergence of the DQM for $\theta=30^{\circ}$ is higher than the one for $\theta=45^{\circ}$. This indicates that the anisotropy of the $\left(45^{\circ} /-45^{\circ} / 45^{\circ}\right)$ square plates is higher than the one of the $\left(30^{\circ} /-30^{\circ} / 30^{\circ}\right)$ square plates for the same material and the anisotropy of the graphite/epoxy square plates with $\left(45^{\circ} /-45^{\circ} / 45^{\circ}\right)$ is the highest.

To ensure the high accuracy of solutions, the frequency parameters of three-layer angle-ply $(\theta /-\theta / \theta)$ square plates with all edges simply supported are obtained by the modified DQM with $31 \times 31$ grid points and are presented in Tables $5-7$. The DQ solutions contain results using two sets of equivalent material constants listed in Table 1 and are all below the upper bound solutions cited from [3]. Note that the Ritz data reported in [3] are exact only for the case of $\theta=0^{\circ}$.

In Table 5, Table 6 , and Table 7, the exact solutions for $\theta=0^{\circ}$ are re-computed by using Eq. (16) with the corresponding material constants, since the existing exact solutions are only 
accurate to two places of decimals. It is observed that the DQ data are exactly the same as the re-computed exact solutions. The exact solutions with MS-I of materials E/E and G/E are slightly higher than the corresponding ones with MS-II, and the exact solutions with MS-I of material B/E are slightly lower than the corresponding ones with MS-II. This trend remains the same in the DQ solutions for other fiber orientation angles. It seems that this trend is mainly caused by the difference of $G_{12}$, since $G_{12}$ in MS-I of materials $\mathrm{E} / \mathrm{E}$ and $\mathrm{G} / \mathrm{E}$ is also slightly larger than $G_{12}$ in MS-II and $G_{12}$ in MS-I of material B/E is smaller than $G_{12}$ in MS-II.

Table 3. Convergence of frequency parameters for angle-ply $\left(30^{\circ} /-30^{\circ} / 30^{\circ}\right)$ SSSS square plates (MS-I)

\begin{tabular}{|c|c|c|c|c|c|c|c|c|c|}
\hline \multirow{2}{*}{ Material } & $N$ & \multicolumn{7}{|c|}{ Mode numbers } \\
\cline { 2 - 9 } & & 1 & 2 & 3 & 4 & 5 & 6 & 7 & 8 \\
\hline \multirow{5}{*}{ E/E } & 11 & 15.8619 & 35.8018 & 42.5515 & 61.3169 & 71.6273 & 85.6521 & 93.5636 & 108.7378 \\
\cline { 2 - 9 } & 15 & 15.8621 & 35.8021 & 42.5519 & 61.3176 & 71.6287 & 85.6529 & 93.5625 & 108.7262 \\
\cline { 2 - 9 } & 19 & 15.8621 & 35.8021 & 42.5520 & 61.3177 & 71.6288 & 85.6529 & 93.5626 & 108.7263 \\
\cline { 2 - 9 } & 23 & 15.8622 & 35.8021 & 42.5520 & 61.3177 & 71.6289 & 85.6530 & 93.5626 & 108.7264 \\
\cline { 2 - 9 } & 27 & 15.8622 & 35.8021 & 42.5520 & 61.3177 & 71.6289 & 85.6530 & 93.5626 & 108.7264 \\
\cline { 2 - 8 } & {$[3]$} & 15.90 & 35.86 & 42.62 & 61.45 & 71.71 & 85.72 & 93.74 & 108.9 \\
\hline \multirow{5}{*}{$\mathrm{B} / \mathrm{E}$} & 11 & 11.9625 & 22.4074 & 35.4364 & 37.4339 & 49.2075 & 55.9908 & 70.5661 & 73.0071 \\
\cline { 2 - 9 } & 15 & 11.9648 & 22.4100 & 35.4424 & 37.4329 & 49.2104 & 55.9665 & 70.4988 & 72.9975 \\
\cline { 2 - 9 } & 19 & 11.9655 & 22.4109 & 35.4444 & 37.4329 & 49.2123 & 55.9665 & 70.4998 & 72.9982 \\
\cline { 2 - 8 } & 23 & 11.9658 & 22.4112 & 35.4453 & 37.4329 & 49.2132 & 55.9665 & 70.5002 & 72.9986 \\
\cline { 2 - 9 } & 27 & 11.9659 & 22.4114 & 35.4457 & 37.4329 & 49.2136 & 55.9665 & 70.5005 & 72.9988 \\
\cline { 2 - 8 } & {$[3]$} & 12.21 & 22.78 & 35.86 & 37.90 & 50.04 & 56.70 & 71.36 & 73.57 \\
\hline \multirow{5}{*}{ G/E } & 11 & 11.6857 & 21.5346 & 35.4172 & 35.5276 & 48.6468 & 52.6563 & 69.1293 & 71.4666 \\
\cline { 2 - 9 } & 15 & 11.6894 & 21.5392 & 35.4255 & 35.5259 & 48.6519 & 52.6272 & 69.0619 & 71.4086 \\
\cline { 2 - 8 } & 19 & 11.6906 & 21.5407 & 35.4286 & 35.5259 & 48.6552 & 52.6272 & 69.0637 & 71.4088 \\
\cline { 2 - 9 } & 23 & 11.6911 & 21.5414 & 35.4299 & 35.5259 & 48.6569 & 52.6272 & 69.0645 & 71.4089 \\
\cline { 2 - 8 } & 27 & 11.6914 & 21.5417 & 35.4306 & 35.5259 & 48.6576 & 52.6272 & 69.0649 & 71.4090 \\
\cline { 2 - 8 } & {$[3]$} & 11.97 & 21.97 & 35.88 & 36.04 & 49.60 & 53.43 & 70.04 & 72.35 \\
\hline
\end{tabular}

Table 4. Convergence of frequency parameters for angle-ply $\left(45^{\circ} /-45^{\circ} / 45^{\circ}\right)$ SSSS square plates (MS-I)

\begin{tabular}{|c|c|c|c|c|c|c|c|c|c|}
\hline \multirow{2}{*}{ Material } & \multirow{2}{*}{$N$} & \multicolumn{8}{|c|}{ Mode numbers } \\
\hline & & 1 & 2 & 3 & 4 & 5 & 6 & 7 & 8 \\
\hline \multirow{6}{*}{$\mathrm{E} / \mathrm{E}$} & 11 & 16.0871 & 36.8624 & 41.7104 & 61.6715 & 76.9472 & 79.877 & 94.4474 & 108.7482 \\
\hline & 15 & 16.0876 & 36.8626 & 41.7116 & 61.6726 & 76.9474 & 79.880 & 94.4454 & 108.7347 \\
\hline & 19 & 16.0877 & 36.8627 & 41.7120 & 61.6728 & 76.9474 & 79.881 & 94.4456 & 108.7352 \\
\hline & 23 & 16.0878 & 36.8627 & 41.7121 & 61.6728 & 76.9474 & 79.881 & 94.4456 & 108.7354 \\
\hline & 27 & 16.0878 & 36.8627 & 41.7121 & 61.6728 & 76.9474 & 79.881 & 94.4456 & 108.7355 \\
\hline & [3] & 16.14 & 36.93 & 41.81 & 61.85 & \begin{tabular}{|l|}
77.04 \\
\end{tabular} & 80.00 & 94.68 & \begin{tabular}{l|l|} 
& 109.0 \\
\end{tabular} \\
\hline \multirow{6}{*}{$\mathrm{B} / \mathrm{E}$} & 11 & 12.3054 & 24.1007 & 33.5834 & 39.5290 & 53.7288 & 58.347 & 64.9806 & 76.8154 \\
\hline & 15 & 12.3196 & 24.1000 & 33.6269 & 39.5290 & 53.7162 & 58.320 & 65.0412 & 76.7477 \\
\hline & 19 & 12.3253 & 24.0999 & 33.6443 & 39.5297 & 53.7162 & 58.319 & 65.0666 & 76.7506 \\
\hline & 23 & 12.3281 & 24.0999 & 33.6528 & 39.5301 & 53.7162 & 58.319 & 65.0794 & 76.7522 \\
\hline & 27 & 12.3298 & 24.0999 & 33.6576 & 39.5303 & 53.7162 & 58.319 & 65.0868 & 76.7532 \\
\hline & [3] & 12.71 & 24.51 & 34.44 & 40.23 & 54.44 & 59.40 & 66.38 & 78.00 \\
\hline \multirow{6}{*}{ G/E } & 11 & 11.8647 & 23.2991 & 33.2088 & 37.7016 & 53.3682 & 55.200 & 64.6746 & 75.3103 \\
\hline & 15 & 11.8774 & 23.2987 & 33.2480 & 37.7018 & 53.3533 & 55.170 & 64.7192 & 75.2411 \\
\hline & 19 & 11.8824 & 23.2987 & 33.2641 & 37.7028 & 53.3533 & 55.170 & 64.7400 & 75.2449 \\
\hline & 23 & 11.8850 & 23.2987 & 33.2720 & 37.7033 & 53.3533 & 55.170 & 64.7507 & 75.2470 \\
\hline & 27 & 11.8865 & 23.2987 & 33.2765 & 37.7036 & 53.3533 & 55.170 & 64.7571 & 75.2482 \\
\hline & [3] & 12.31 & 23.72 & 34.14 & 38.45 & 54.10 & 56.31 & 66.20 & 76.23 \\
\hline
\end{tabular}


Table 5. Frequency parameters of angle-ply $(\theta /-\theta / \theta)$ SSSS square plates (E/E, $N=31)$

\begin{tabular}{|c|c|c|c|c|c|c|c|c|c|}
\hline \multirow{2}{*}{$\theta^{\circ}$} & \multirow{2}{*}{ Methods } & \multicolumn{8}{|c|}{ Mode numbers } \\
\cline { 3 - 10 } & & 1 & 2 & 3 & 4 & 5 & 6 & 7 & 8 \\
\hline \multirow{4}{*}{0} & DQM (I) & 15.19467 & 33.29959 & 44.41877 & 60.77869 & 64.52979 & 90.30141 & 93.66415 & 108.5563 \\
\cline { 2 - 10 } & Exact (I) & 15.19467 & 33.29959 & 44.41877 & 60.77869 & 64.52979 & 90.30141 & 93.66415 & 108.5563 \\
\cline { 2 - 10 } & DQM (II) & 15.17055 & 33.24847 & 44.38711 & 60.68220 & 64.45675 & 90.14548 & 93.63063 & 108.4588 \\
\cline { 2 - 10 } & Exact (II) & 15.17055 & 33.24847 & 44.38711 & 60.68220 & 64.45675 & 90.14548 & 93.63063 & 108.4588 \\
\hline \multirow{3}{*}{15} & DQM (I) & 15.4150 & 34.0748 & 43.8514 & 60.8068 & 66.6413 & 91.3847 & 91.5001 & 108.8889 \\
\cline { 2 - 10 } & {$[3]$} & 15.43 & 34.09 & 43.87 & 60.85 & 66.67 & 91.40 & 91.56 & 108.9 \\
\cline { 2 - 10 } & DQM (II) & 15.3959 & 34.0299 & 43.8199 & 60.7327 & 66.5601 & 91.3403 & 91.3773 & 108.7845 \\
\hline \multirow{3}{*}{30} & DQM (I) & 15.8622 & 35.8021 & 42.5521 & 61.3177 & 71.6289 & 85.6530 & 93.5627 & 108.7265 \\
\cline { 2 - 10 } & {$[3]$} & 15.90 & 35.86 & 42.62 & 61.45 & 71.71 & 85.72 & 93.74 & 108.9 \\
\hline & DQM (II) & 15.8534 & 35.7679 & 42.5238 & 61.2745 & 71.5463 & 85.5891 & 93.4889 & 108.6531 \\
\hline \multirow{3}{*}{45} & DQM (I) & 16.0880 & 36.8627 & 41.7122 & 61.6729 & 76.9474 & 79.8813 & 94.4456 & 108.7356 \\
\cline { 2 - 9 } & {$[3]$} & 16.14 & 36.93 & 41.81 & 61.85 & 77.04 & 80.00 & 94.68 & 109.0 \\
\cline { 2 - 9 } & DQM (II) & 16.0842 & 36.8321 & 41.6880 & 61.6430 & 76.8622 & 79.8129 & 94.3878 & 108.6515 \\
\hline
\end{tabular}

Table 6. Frequency parameters of angle-ply $(\theta /-\theta / \theta)$ SSSS square plates $(\mathrm{B} / \mathrm{E}, N=31)$

\begin{tabular}{|c|c|c|c|c|c|c|c|c|c|}
\hline \multirow{2}{*}{$\theta^{\circ}$} & \multirow{2}{*}{ Methods } & \multicolumn{7}{|c|}{ Mode numbers } \\
\cline { 2 - 11 } & & 1 & 2 & 3 & 4 & 5 & 6 & 7 & 8 \\
\hline \multirow{4}{*}{0} & DQM (I) & 11.03935 & 17.36394 & 30.90502 & 40.37093 & 44.15742 & 51.12759 & 53.26851 & 69.45577 \\
\cline { 2 - 10 } & Exact (I) & 11.03935 & 17.36394 & 30.90502 & 40.37093 & 44.15742 & 51.12759 & 53.26851 & 69.45577 \\
\cline { 2 - 10 } & DQM (II) & 11.04440 & 17.37677 & 30.92123 & 40.37645 & 44.17759 & 51.14502 & 53.30614 & 69.50708 \\
\cline { 2 - 10 } & Exact (II) & 11.04440 & 17.37677 & 30.92123 & 40.37645 & 44.17759 & 51.14502 & 53.30614 & 69.50708 \\
\hline \multirow{4}{*}{15} & DQM (I) & 11.3047 & 19.0789 & 33.1642 & 38.7790 & 45.2024 & 51.9267 & 59.1244 & 72.3957 \\
\cline { 2 - 10 } & {$[3]$} & 11.37 & 19.21 & 33.32 & 38.86 & 45.46 & 52.14 & 59.48 & 72.77 \\
\cline { 2 - 10 } & DQM (II) & 11.3089 & 19.0890 & 33.1790 & 38.7854 & 45.2210 & 51.9463 & 59.1566 & 72.4253 \\
\hline \multirow{3}{*}{30} & DQM (I) & 11.9660 & 22.4115 & 35.4460 & 37.4329 & 49.2139 & 55.9665 & 70.5006 & 72.9989 \\
\cline { 2 - 9 } & {$[3]$} & 12.21 & 22.78 & 35.86 & 37.90 & 50.04 & 56.70 & 71.36 & 73.57 \\
\cline { 2 - 10 } & DQM (II) & 11.9678 & 22.4180 & 35.4527 & 37.4446 & 49.2295 & 55.9816 & 70.5315 & 73.0123 \\
\hline \multirow{3}{*}{45} & DQM (I) & 12.3308 & 24.0999 & 33.6606 & 39.5305 & 53.7162 & 58.3197 & 65.0916 & 76.7538 \\
\cline { 2 - 9 } & {$[3]$} & 12.71 & 24.51 & 34.44 & 40.23 & 54.44 & 59.40 & 66.38 & 78.00 \\
\cline { 2 - 9 } & DQM (II) & 12.3315 & 24.1065 & 33.6659 & 39.5399 & 53.7361 & 58.3325 & 65.1028 & 76.7794 \\
\hline
\end{tabular}

Table 7. Frequency parameters of angle-ply $(\theta /-\theta / \theta)$ SSSS square plates $(\mathrm{G} / \mathrm{E}, N=31)$

\begin{tabular}{|c|c|c|c|c|c|c|c|c|c|}
\hline \multirow{2}{*}{$\theta^{\circ}$} & \multirow{2}{*}{ Methods } & \multicolumn{8}{|c|}{ Mode } \\
\cline { 3 - 11 } & & 1 & 2 & 3 & 4 & 5 & 6 & 7 & 8 \\
\hline \multirow{4}{*}{0} & DQM (I) & 11.28972 & 17.13178 & 28.69169 & 40.74023 & 45.15887 & 45.78291 & 54.08234 & 68.14209 \\
\cline { 2 - 10 } & Exact (I) & 11.28972 & 17.13178 & 28.69169 & 40.74023 & 45.15887 & 45.78291 & 54.08234 & 68.14209 \\
\cline { 2 - 10 } & DQM (II) & 11.28718 & 17.12536 & 28.68364 & 40.73740 & 45.14874 & 45.77484 & 54.06362 & 68.13483 \\
\cline { 2 - 10 } & Exact (II) & 11.28718 & 17.12536 & 28.68364 & 40.73740 & 45.14874 & 45.77484 & 54.06362 & 68.13483 \\
\hline \multirow{4}{*}{15} & DQM (I) & 11.3927 & 18.5447 & 31.0178 & 39.0659 & 45.6444 & 47.9654 & 58.2869 & 67.4789 \\
\cline { 2 - 10 } & {$[3]$} & 11.46 & 18.69 & 31.20 & 39.15 & 45.91 & 48.19 & 58.70 & 67.84 \\
\cline { 2 - 9 } & DQM (II) & 11.3906 & 18.5398 & 31.0109 & 39.0628 & 45.6354 & 47.9567 & 58.2711 & 67.4670 \\
\hline \multirow{3}{*}{30} & DQM (I) & 11.6916 & 21.5420 & 35.4311 & 35.5259 & 48.6582 & 52.6273 & 69.0651 & 71.4091 \\
\cline { 2 - 9 } & {$[3]$} & 11.97 & 21.97 & 35.88 & 36.04 & 49.60 & 53.43 & 70.04 & 72.35 \\
\cline { 2 - 9 } & DQM (II) & 11.6908 & 21.5389 & 35.4278 & 35.5204 & 48.6507 & 52.6203 & 69.0502 & 71.4019 \\
\hline \multirow{3}{*}{45} & DQM (I) & 11.8875 & 23.2987 & 33.2794 & 37.7038 & 53.3533 & 55.1707 & 64.7612 & 75.2490 \\
\cline { 2 - 9 } & {$[3]$} & 12.31 & 23.72 & 34.14 & 38.45 & 54.10 & 56.31 & 66.20 & 76.63 \\
\cline { 2 - 9 } & DQM (II) & 11.8873 & 23.2956 & 33.2768 & 37.6996 & 53.3432 & 55.1652 & 64.7556 & 75.2369 \\
\hline
\end{tabular}

\section{Conclusions}

The free vibration of mid-plane symmetric angle-ply laminated composite square plates with all edges simply supported is successfully solved by using the modified differential quadrature 
method (modified DQM). Three material systems are considered. The rate of convergence of the modified DQM is investigated. The results are tabulated for references.

Based on the results reported herein, one may conclude that the DQ data are highly accurate and can be served as the benchmark solutions. The difference in solutions of the mid-plane symmetric angle-ply laminated composite plates with two sets of equivalent material constants is clearly seen and thus care should be taken when highly accurate results are needed for comparisons in testing newly developed numerical methods. However, the difference is small and negligible from the practical point of view.

\section{Acknowledgement}

The project is partially supported by the Priority Academic Program Development of Jiangsu Higher Education Institutions.

\section{References}

[1] Whitney J. M. Structural Analysis of Laminated Anisotropic Plates. Technomic Publishing Company Inc, Pennsylvania, 1987.

[2] Ovesy H. R., Ghannadpour S. A. M., Sherafat M. H. Buckling analysis of laminated composite plates using higher order semi-analytical finite strip method. Applied Composite Materials, Vol. 17, 2010, p. 69-80.

[3] Leissa A. W., Narita Y. Vibration studies for simply supported symmetrically laminated rectangular plates. Composite Structures, Vol. 12, 1989, p. 113-132.

[4] Chow S. T., Liew K. M., Lam K. Y. Transverse vibration of symmetrically laminated rectangular composite plates. Composite Structures, Vol. 20, 1992, p. 213-226.

[5] Hashemi S. M., Roach A. Dynamic finite element analysis of extensonal-tortional coupled vibration in nonuniform composite beams. Applied Composite Materials, Vol. 18, 2011, p. 521-538.

[6] Seçgin A., Sarıgül A. S. Free vibration analysis of symmetrically laminated thin composite plates by using discrete singular convolution (DSC) approach: algorithm and verification. Journal of Sound and Vibration, Vol. 315, Issues 1-2, 2008, p. 197-211.

[7] Zhu Q., Wang X. Free vibration analysis of thin isotropic and anisotropic rectangular plates by the discrete singular convolution algorithm. International Journal for Numerical Methods in Engineering, Vol. 86, 2011, p. 782-800.

[8] Wang X., Wang Y., Xu S. DSC analysis of a simply supported anisotropic rectangular plate. Composite Structures, Vol. 94, 2012, p. 2576-2584.

[9] Wang X. Differential Quadrature and Differential Quadrature Based Element Methods: Theory and Applications. Butterworth-Heinemann, Oxford, 2015.

[10] Messina A. Analyses of freely vibrating cross-ply laminated plates in conjunction with adaptive global piecewise-smooth functions (A-GPSFs). International Journal of Mechanical Sciences, Vol. 90, 2015, p. $179-189$.

[11] Stone M. A., Chandler H. D. Errors in double sine series solutions for simply supported symmetrically laminated plates. International Journal of Mechanical Sciences, Vol. 38, Issue 5, 1996, p. 517-526.

[12] Vinson J. R., Chou T. W. Composite Materials and Their Use in Structures. Applied Science Publisher Ltd, London, 1975. 